
\title{
3 Research Square \\ High Expression of PABPC1 Predicts Worse Survival of Gastric Cancer Patients
}

\section{Tailai An}

The Seventh Affiliated Hospital Sun Yat-sen University https://orcid.org/0000-0003-0666-752X

\section{Lingna Deng}

The Seventh Affiliated Hospital Sun Yat-sen University

\section{Zheng Yang}

The Seventh Affiliated Hospital Sun Yat-sen University

\section{Cuicui Chai}

The Seventh Affiliated Hospital Sun Yat-sen University

\section{Yan Wang}

Shenzhen People's Hospital

\section{Jun Ouyang}

The Seventh Affiliated Hospital Sun Yat-sen University

\section{Xiaofang Lu}

The Seventh Affiliated Hospital Sun Yat-sen University

Changhua Zhang ( $\square$ zhchangh@mail.sysu.edu.cn )

The Seventh Affiliated Hospital Sun Yat-sen University

\section{Research}

Keywords: Gastric cancer, PABPC1, Overall survival, Disease-free survival

Posted Date: November 3rd, 2020

DOI: https://doi.org/10.21203/rs.3.rs-99696/v1

License: (c) (1) This work is licensed under a Creative Commons Attribution 4.0 International License. Read Full License 


\section{Abstract}

Background: Gastric cancer (GC) is one of the most common cancers with one of the highest mortality rates. Unfortunately, underlying molecular mechanisms contributing to GC have not been fully illuminated. PABPC1 is involved in a series of processes, such as mRNA translation, and mRNA deadenylation and decay. We performed this study to clarify the role of PABPC1 in GC.

Methods: To evaluate PABPC1 expressions in GC and normal tissues, we performed bioinformatics analysis of data from TCGA. PABPC1 expressions were evaluated by immunohistochemical (IHC) staining of $170 \mathrm{GC}$ specimens. Associations between PABPC1 expression and clinicopathological variables were analyzed. Independent predictive factors for survival of GC patients were determined by Cox regression analysis.

Results: It was revealed by bioinformatics analysis that compared with normal gastric tissues, PABPC1 expressions in $G C$ tissues were significantly higher ( $P=0.002$, paired) $\left(P=3.605 \mathrm{e}^{\wedge-9}\right.$, unpaired). It was revealed that $P A B P C 1$ expression was significantly associated with tumor size $(P=0.008)$, Borrmann classification ( $P=0.003)$, vessel invasion $(P=0.017)$, depth of invasion $(P=0.032)$, lymph node metastasis $(P=0.001)$, and TNM stage $(P=0.019)$. It was demonstrated through Cox regression analysis that PABPC1 expression was a predictive factor for both overall survival $(O S)(P<0.001)$ and disease-free survival (DFS) $(P<0.001)$ of GC patients.

Conclusions: Compared with that of normal gastric tissue, expression level of PABPC1 in GC tissue was significantly higher and PABPC1's high expression was significantly associated with poorer survival, suggesting its potential as a therapeutic biomarker for GC.

\section{Background}

As one of the most common and most deadly malignant tumors, gastric cancer (GC) is quite prevalent in China as nearly half of all the GC patients are diagnosed in China[1, 2]. The long-term outcome of patients with GC are still rather poor even if we have made much progress in diagnosis and treatment of GC patients over the recent years. For patients with resectable GC, curative gastrectomy combined with subsequent adjuvant treatment is still the first choice. Unfortunately, most GC patients experience recurrences after curative surgery, which is the main cause of shortened survival of GC patients. As the most important cause for cancer-related deaths, metastasis is a complex, multistep process that involves multiple signaling pathway[3-5]. Thus, it is still necessary for us to explore novel therapeutic biomarkers for GC.

As proteins that interact with single-stranded poly $(A)$ by RNA recognition motif, polyadenylate binding proteins (PABPs) were classified into polyadenylate binding protein nucleus (PABPN) located in the nucleus and polyadenylate binding protein cytoplasm (PABPC) located in cytoplasm. It has been reported in many studies that PABPCs are involved in many processes such as translation, control of the rate of mRNA deadenylation and mRNA decay [6-9]. PABPs have been reported to participate in multiple 
physiological processes. In a study published in 2001, it was reported that in spermatids, the expression of PABP3 might be utilized as an alternative to bypass PABP1 translational repression and to produce the amount of PABP needed for active mRNA translation[10]. It was then reported by Akiko Yanagiya et al that PAIP2A-PABP interaction influenced the production of essential proteins for germ cell maturation during late spermiogenesis[11] Yang $\mathrm{H}$ et al reported the up-regulation of PABPC4 relied on cell status, such as during $T$ cell activation[12]. It was reported that nuclear relocation of cytoplasmic PABP1 and PABP4 caused by UV irradiation revealed mRNA-dependent export of metazoan PABPS [13]. Encoded on the $X$ chromosome, PABPC5 was expressed fetal brain and multiple adult tissues [14]. PABPs have also been reported to be involved in carcinogenesis, which is no surprise at all since mechanisms responsible for mRNA degradation control gene expression. PABPC4 expression has been reported to regulate telomerase activity and proliferation of cervical cancer cell lines[15], to be associated with prognosis of colorectal cancer patients[16], and to promote cancer stem cell features of hepatocellular carcinoma[17]. Insufficient Papi2 level caused by knockdown of PABP led to decreased mRNA translation and deaths of the Hela cell lines[18].

As the most typical polyadenylate binding protein, PABPC1 is universally expressed in eukaryotes[19]. Many previous studies have reported that PABPC1 is involved in esophageal cancer, breast cancer, prostate cancer, colorectal cancer and hepatocellular carcinoma. In esophageal cancer, decreased expression of PABPC1 was associated with tumor progression and poor survival[20]. Dong $\mathrm{H}$ et al reported that long non-coding RNA SNHG14 induces trastuzumab resistance of breast cancer via regulating PABPC1 expression through H3K27 acetylation[21]. Whereas in prostate cancer, PABPC1 has been demonstrated to be associated with aggressive phenotype[22]. Wu YQ reported that PABPC1L depletion inhibits proliferation and migration via blockage of AKT pathway in human colorectal cancer cells[23]. In hepatocellular carcinoma, it was reported that IncRNA SNHG14 promoted hepatocellular carcinoma progression via H3K27 acetylation-activated PABPC1 by PTEN signaling[24] and PABPC1,s interaction with AGO2 and is responsible for the micr[20]oRNA mediated gene silencing in high grade hepatocellular carcinoma[25].

However, the role and mechanisms of PABPC1 in gastric cancer is by far still ambiguous. In a study published in 2015, PABPC1 was reported to exert carcinogenesis and promote growth and survival of gastric cancer cells by regulating miR-34c [26]. Despite it was also reported that high expression of PABPC1 was significantly associated with poorer survival of patients with GC, Cox regression analysis was not performed to identify independent prognostic factors and figures demonstrating the results of IHC staining were not presented. Thus, we perform this study to assess the expression level of PABPC1 in GC tissue and the association between PABPC1 expression and survival of GC patients.

\section{Materials And Methods}

\section{Study population and data collection}


Our study had gained approval from the Ethics Committee of The Seventh Affiliated Hospital, Sun Yat-sen University. Informed consent in written form was obtained from each individual patient.

Clinicopathological information of the patients were retrospectively collected and screened. From January 2009 to December 2013, these patients had undergone curative surgery at the First Affiliated Hospital, Sun Yat-sen University and been histopathologically diagnosed with GC. All the follow-ups were terminated on December 31st, 2019. In all, 170 GC patients with complete clinicopathological information were enrolled in our study. During the whole process, we performed the present study in compliance with the Declaration of Helsinki. Clinicopathological data of the 170 patients including age, gender, operation type, tumor size, Bormann classification, histological differentiation, depth of invasion, lymph node metastasis, TNM stage, CEA level, and vessel or nerve invasion were collected from medical archives. TNM stages were determined according to the eighth edition of American Joint Committee on Cancer(AJCC)/International Union Against Cancer TNM classification system.

\section{Bioinformatics analysis}

Relevant data were downloaded from The Cancer Genome Atlas (TCGA) database (https://cancergenome.nih.gov) and statistical analyses of these data were performed to assess the expression of PABPC1 in GC. Comparison between the expression levels of PABPC1 in GC tissues and those in normal gastric tissues were also made according to the TCGA database.

\section{Immunohistochemical staining and scoring}

In order to quantify the expression of PABPC1 in GC tissues, we performed immunohistochemical staining using the slides of $170 \mathrm{GC}$ patients. Initially, the GC tissues were embedded with paraffin immediately after being removed. After being deparaffinized with xylene, GC slides were then rehydrated with alcohol of different concentrations ( $100 \%, 95 \%, 85 \%$, and $75 \%) .0 .3 \%$ hydrogen peroxide was used to block endogenous peioxidase activity of GC tissues (soaking the slides in $0.3 \%$ hydrogen peroxide for 20 minutes). The the slides were blocked with $10 \%$ BSA. After being blocked by $10 \%$ bovine serum albumin (BSA), the slides were incubated with PABPC1-specific antibodies (66809-1-Ig, 1:800, proteintech group, Wuhan, Hubei, China) at 4०C overnight. On the next day, the slides were incubated with secondary antibodies (GK600510, Gene Tech, Shanghai, China) at room temperature for half an hour. Then the antibody-conjugated PABPC1 was visualized with 3,3,-diaminobenzidine (DAB) (GK600510, Gene Tech, Shanghai, China). Subsequently, the DAB-stained slides were counter stained with hematoxylin. Ultimately, the images were captured using an inverted microscope (Olympus, BX53, Japan). Two experienced pathologists without knowing the clinicopathological information of the enrolled patients independently scored the stained slides. Brown-yellow staining was indicative of PABPC1-positivity. It was by multiplying staining intensity and percentage of positive cells that the total scores of the GC slides were calculated. Staining intensity was defined as follows: no staining (0) (shown in Fig. 1a and Fig. 1e), weak staining (1) (shown in Fig. 1b and Fig. 1f), moderate staining(2) (shown in Fig. 1c and Fig. 1g), and strong staining(3) (shown in Fig. $1 \mathrm{~d}$ and Fig. 1h). Whereas, percentage of positive cells was defined according to the following criteria: $1(1 \%-25 \%), 2(26 \%-50 \%), 3(51 \%-75 \%)$, and $4(76 \%-100 \%)$. Total 
score $\geq 6$ was used to define as high expression while low expression was recorded when it was less than 6.

\section{Statistical analysis}

The associations between the expression level of PABPC1 and clinicopathological variables of the 170 GC patients were assessed by Chi square test and Fisher's exact test. The survival of GC patients were calculated by the Kaplan-Meier Method and tested via the log-rank test. The time span between curative gastrectomy and death was defined as overall survival (OS) while the duration between curative surgery and the first recurrence or death (whichever occurred first) was named as disease-free survival (DFS). The independent predictive factors for OS and DFS were verified by performing Cox regression analysis (both univariate and multivariate). SPSS22 (Chicago, IL, USA) was used to perform all the statistical analyses in the present study. All tests in this study were two-sided in nature and statistical significance was recorded when the $P$ value was $<0.05$.

\section{Results}

\section{Baseline characteristics of GC patients}

Baseline characteristics of the 170 enrolled GC patients and the relations between the expression of PABPC1 and clinicopathological variables were summarized and shown in Table 1. It was revealed that the expression of PABPC1 was significantly associated with tumor size $(P=0.008)$, Bormann classification $(P=0.003)$, vessel invasion $(P=0.017)$, depth of invasion $(P=0.032)$, lymph node metastasis $(P=0.001)$, and TNM stage $(P=0.019)$.

\section{Bioinformatics analysis}

The heatmap of bioinformatics analyses revealed that many genes were up-regulated or down-regulated in $\mathrm{GC}$ and PABPC1 belonged to the former ones (shown in Fig. 2a). In order to evaluate the expression levels of PABPC1 in GC tissues, we analyzed the downloaded RNA-seq data, the results of which demonstrated that in comparison with those in normal gastric tissues, the expression levels of PABPC1 in GC tissues were significantly higher $(P=0.002$, paired, shown in Fig. $2 b)\left(P=3.605 \mathrm{e}^{\wedge-9}\right.$, unpaired, shown in Fig. 2c). Thus, we could conclude that PABPC1 expression level was significantly up-regulated in GC tissues.

\section{Survival analysis}

The median survival time of the 170 included GC patients was 39 months. Kaplan-Meier analysis revealed that the expression level of PABPC1 was significantly associated with $\mathrm{OS}(P<0.001)$ (shown in Fig. 3a) and DFS $(P<0.001)$ (shown in Fig. 3b). Univariate Cox regression analysis revealed that tumor size $(P=0.021, \mathrm{HR}=2.464,95 \% \mathrm{Cl}: 1.543-3.935)$, Bormman classification $(\mathrm{P}<0.001, \mathrm{HR}=4.388,95 \% \mathrm{Cl}$ : 2.358-8.168), differentiation ( $P=0.028, H R=1.771,95 \% \mathrm{Cl}: 1.063-2.953)$, depth of invasion $(P<0.001$, $\mathrm{HR}=1.874,95 \% \mathrm{Cl}: 1.519-2.313)$, lymph node metastasis $(\mathrm{P}<0.001, \mathrm{HR}=2.290,95 \% \mathrm{Cl}: 1.887-2.779)$, 
TNM stage $(P<0.001, H R=3.620,95 \%$ Cl: 2.369-5.531), CEA level $(P=0.007, H R=2.228,95 \%$ Cl: $1.240-$ 4.004), vessel invasion ( $P=0.031, H R=3.365,95 \% C l: 2.018-5.612)$, nerve invasion $(P=0.008, H R=$ 2.522, 95\% Cl: 1.278-4.977), and PABPC1 expression ( $\mathrm{P}<0.001, \mathrm{HR}=3.643,95 \% \mathrm{Cl}: 2.149-6.176)$ were significantly associated OS (shown in Table 2). Subsequently, these variables significantly associated with OS proven by univariate Cox regression analysis were included in multivariate Cox regression analysis, the results of which revealed that depth of invasion $(P=0.048, H R=1.400,95 \% \mathrm{Cl}: 1.001-1.958)$, lymph node metastasis $(P=0.012, H R=1.796,95 \% \mathrm{Cl}: 1.342-2.405)$, pTNM $(P=0.027, H R=2.875,95 \%$ Cl: 1.969-3.319) and PABPC1 expression ( $\mathrm{P}=0.013, \mathrm{HR}=2.058,95 \% \mathrm{Cl}: 1.162-3.644)$ were independent prognostic factors for OS (shown in Table 2).

Similarly, through univariate Cox regression analysis, Bormann classification $(P=0.009, H R=3.892,95 \%$ Cl: 1.880-8.060), differentiation $(P=0.011, \mathrm{HR}=2.553,95 \% \mathrm{Cl}: 1.236-5.271)$, depth of invasion $(P<$ $0.001, \mathrm{HR}=1.858,95 \% \mathrm{Cl}: 1.442-2.395)$, lymph node metastasis $(\mathrm{P}=0.038, \mathrm{HR}=2.194,95 \% \mathrm{Cl}: 1.735-$ 2.774), TNM stage $(P<0.001, H R=3.761,95 \% \mathrm{Cl}: 2.230-6.342), \mathrm{CEA}$ level $(\mathrm{P}=0.044, \mathrm{HR}=2.115,95 \% \mathrm{Cl}$ : 1.021-4.380), vessel invasion $(P=0.008, H R=4.892,95 \% \mathrm{Cl}: 2.667-8.972)$, nerve invasion $(P=0.042, H R$ $=2.911,95 \% \mathrm{Cl}: 1.349-6.282)$ and PABPC1 expression ( $\mathrm{P}=0.008, \mathrm{HR}=3.450,95 \% \mathrm{Cl}: 1.813-6.564)$ were proven to be significantly associated with DFS. Subsequently, the variables significantly associated with DFS proven by univariate Cox regression analysis were included in multivariate Cox regression analysis, the results of which revealed that vessel invasion $(P=0.010, \mathrm{HR}=2.700,95 \% \mathrm{Cl}: 1.265-5.764), \mathrm{pTNM}(\mathrm{P}$ $=0.021, \mathrm{HR}=3.874,95 \% \mathrm{Cl}: 2.649-4.434)$, and PABPC1 expression ( $\mathrm{P}=0.018, \mathrm{HR}=2.284,95 \% \mathrm{Cl}: 1.153-$ 4.524) were independent prognostic predictors for DFS of patients with GC (shown in Table 3).

\section{Discussion}

It was revealed in the present study that overexpression of PABPC1 was significantly associated with poor survival of GC patients and it had the potential as a prognostic biomarker for patients with GC. Firstly, data from TCGA database were analyzed, demonstrating that the mRNA levels of PABPC1 in GC tissues were significantly higher than those in normal gastric tissues. Secondly, it was revealed through Kaplan-Meier analysis that high expression of PABPC1 was significantly related with poorer OS and DFS. Thirdly, it was proven by Cox regression analysis that PABPC1 could independently predict OS and DFS of GC patients.

The PABPC gene encodes a cytoplasmic protein interacting with single-stranded poly $(A)$ by RNA recognition motif. PABPs have been reported by a series of studies to participate in a few cellular processes including translation, control of the rate of mRNA deadenylation and mRNA decay[6-9]. Besides being involved in these cellular processes, PABPCs have been reported to be involved in spermiogenesis [10, 11], UV irradiation[13],and fetal organ development[14]. As the most typical polyadenylate binding protein, PABPC1 has been reported to be involved in the pathogenesis of colorectal cancer, esophageal cancer, breast cancer, prostate cancer, and hepatocellular carcinoma. It is no surprise to us at all that PABPC1 plays such important roles in these malignant tumors since PABPC1 controls mRNA metabolisms. 
It was also demonstrated in the present study that expression of PABPC1 was significantly associated with Bormann classification, vessel invasion, depth of invasion, lymph node metastasis, and TNM stage. All these parameters are signs for aggressive phenotype of GC, explaining why high expression of PABPC1 was significantly and negatively associated with OS and DFS. The specific mechanisms through which PABPC1 contributed to GC and poor survival of GC patients are, however, not discussed in the present study due to the limited time schedule, which is the limitation of our study. The roles of PABPC1 in other malignant tumors have been studied. In esophageal cancer, decreased expression of PABPC1 was associated with tumor progression and poor survival [20]. Dong $\mathrm{H}$ et al reported that long non-coding RNA SNHG14 induces trastuzumab resistance of breast cancer via regulating PABPC1 expression through H3K27 acetylation[21]. Whereas in prostate cancer, PABPC1 has been demonstrated to be associated with aggressive phenotype [22]. Wu YQ et al reported that PABPC1L depletion inhibits proliferation and migration via blockage of AKT pathway in human colorectal cancer cells [23]. In hepatocellular carcinoma, it was reported that IncRNA SNHG14 promoted hepatocellular carcinoma progression via H3K27 acetylation-activated PABPC1 by PTEN signaling[24] and PABPC1's interaction with AGO2 and is responsible for the microRNA mediated gene silencing in high grade hepatocellular carcinoma[25].

In conclusion, the expression of PABPC1 in gastric cancer is significantly higher in gastric cancer tissues than that in normal gastric tissues, and its overexpression is significantly associated with poor prognosis, suggesting its potential as a novel biomarker for developing targeted drugs.

\section{Conclusions}

In conclusion, the expression of PABPC1 in gastric cancer is significantly higher in gastric cancer tissues than that in normal gastric tissues, and its overexpression is significantly associated with poor prognosis, suggesting its potential as a novel biomarker for developing targeted drugs.

\section{Abbreviations}

GC: gastric cancer; IHC: immunohistochemical; TCGA: The Cancer Genome Atlas; OS: overall survival; DFS: disease-free survival; PABP: polyadenylate binding protein; PABPN: polyadenylate binding protein nucleus; PABPC: polyadenylate binding protein cytoplasm; CEA: carcinoembryonic antigen; BSA: bovine serum albumin; DAB: 3,3,-diaminobenzidine; HR: hazard ratio; Cl: confidential interval; UV: ultraviolet.

\section{Declarations}

\section{Ethics approval and consent to participate}

Our study was approved by the ethics committee of the Seventh Affiliated Hospital of Sun Yat-sen University and the declaration of Helsinki was complied with. Approval Letter for Research Protocol: No. 339 [2020]. All the patients had given their written informed consent. 
Consent for publication

Not applicable.

\section{Availability of data and materials}

The data analyzed in this study were available from the corresponding authors on reasonable requests.

\section{Competing interests}

The authors declare that they have no competing interests.

\section{Funding}

The present study received support from Shenzhen Key Medical discipline Construction Fund (No.SZXK016).

\section{Authors contribution}

TL A, LJ C, Y W, J OY, and LN D were responsible for data collection, statistical analysis and paper writing. $Y W$ was responsible for radiological examinations. $L N D$ was responsible for proof reading. $X F L$ and TL A were responsible for immunohistochemical staining. $\mathrm{XF} \mathrm{L}$ and $\mathrm{CH} Z$ were responsible for designing and supervising the study.

\section{Acknowledgements}

The authors would thank Junqing Li and Wenjie Zhou from The Seventh Affiliated Hospital, Sun Yat-sen university for their assistance with statistical analysis.

\section{References}

1. Siegel RL, Miller KD, Jemal A: Cancer statistics, 2020. CA Cancer J Clin 2020, 70:7-30.

2. Chen W, Zheng R, Baade PD, Zhang S, Zeng H, Bray F, Jemal A, Yu XQ, He J: Cancer statistics in China, 2015. CA: A Cancer Journal for Clinicians 2016, 66:115-132.

3. Pitroda SP, Weichselbaum RR: Integrated molecular and clinical staging defines the spectrum of metastatic cancer. Nat Rev Clin Oncol 2019, 16:581-588.

4. Massagué J, Batlle E, Gomis RR: Understanding the molecular mechanisms driving metastasis. $\mathrm{Mol}$ Oncol 2017, 11:3-4.

5. Plaks V, Kong N, Werb Z: The cancer stem cell niche: how essential is the niche in regulating stemness of tumor cells? Cell Stem Cell 2015, 16:225-238.

6. Wigington $\mathrm{CP}$, Williams KR, Meers MP, Bassell GJ, Corbett AH: Poly(A) RNA-binding proteins and polyadenosine RNA: new members and novel functions. Wiley Interdiscip Rev RNA 2014, 5:601-622. 
7. Goss DJ, Kleiman FE: Poly(A) binding proteins: are they all created equal? Wiley Interdiscip Rev RNA 2013, 4:167-179.

8. Villalba A, Coll O, Gebauer F: Cytoplasmic polyadenylation and translational control. Curr Opin Genet Dev 2011, 21:452-457.

9. Kini HK, Vishnu MR, Liebhaber SA: Too much PABP, too little translation. J Clin Invest 2010, 120:3090-3093.

10. Féral C, Guellaën G, Pawlak A: Human testis expresses a specific poly(A)-binding protein. Nucleic Acids Res 2001, 29:1872-1883.

11. Yanagiya A, Delbes G, Svitkin YV, Robaire B, Sonenberg N: The poly(A)-binding protein partner Paip2a controls translation during late spermiogenesis in mice. Journal of Clinical Investigation 2010, 120:3389-3400.

12. Yang $H$, Duckett CS, Lindsten T: iPABP, an Inducible Poly(A)-Binding Protein Detected in Activated Human T Cells. Mol Cell Biol 1995, 15:6770-6776.

13. Burgess HM, Richardson WA, Anderson RC, Salaun C, Graham SV, Gray NK: Nuclear relocalisation of cytoplasmic poly(A)-binding proteins PABP1 and PABP4 in response to UV irradiation reveals mRNAdependent export of metazoan PABPs. Journal of Cell Science 2011, 124:3344-3355.

14. Blanco P, Sargent CA, Boucher CA, Howell G, Ross M, Affara NA: A novel poly(A)-binding protein gene (PABPC5) maps to an X-specific subinterval in the Xq21.3/Yp11.2 homology block of the human sex chromosomes. Genomics 2001, 74:1-11.

15. Katzenellenbogen RA, Vliet-Gregg P, Xu M, Galloway DA: Cytoplasmic poly(A) binding proteins regulate telomerase activity and cell growth in human papillomavirus type 16 E6-expressing keratinocytes. J Virol 2010, 84:12934-12944.

16. Liu D, Yin B, Wang Q, Ju W, Chen Y, Qiu H, Li J, Peng X, Lu C: Cytoplasmic poly(A) binding protein 4 is highly expressed in human colorectal cancer and correlates with better prognosis. J Genet Genomics 2012, 39:369-374.

17. Gu Y, Wei X, Sun Y, Gao H, Zheng X, Wong LL, Jin L, Liu N, Hernandez B, Peplowska K, et al: miR-1925p Silencing by Genetic Aberrations Is a Key Event in Hepatocellular Carcinomas with Cancer Stem Cell Features. Cancer Res 2019, 79:941-953.

18. Thangima Zannat M, Bhattacharjee RB, Bag J: Depletion of cellular poly (A) binding protein prevents protein synthesis and leads to apoptosis in HeLa cells. Biochem Biophys Res Commun 2011, 408:375-381.

19. Grange T, C M de Sa, Oddos J, Pictet R: Human mRNA polyadenylate binding protein-evolutionary conservation of a nucleic acid binding motif. Nucleic Acids Res 1987, 15:4771-4787.

20. Takashima N, Ishiguro H, Kuwabara Y, Kimura M, Haruki N, Ando T, Kurehara H, Sugito N, Mori R, Fujii Y: Expression and prognostic roles of PABPC1 in esophageal cancer[correlation with tumor progression and postoperative survival. Oncol Rep 2006, 15:667-671.

21. Dong H, Wang W, Mo S, Liu Q, Chen X, Chen R, Zhang Y, Zou K, Ye M, He X, et al: Long non-coding RNA SNHG14 induces trastuzumab resistance of breast cancer via regulating PABPC1 expression 
through H3K27 acetylation. J Cell Mol Med 2018, 22:4935-4947.

22. Schaid DJ, McDonnell SK, FitzGerald LM, DeRycke L, Fogarty Z, Giles GG, Maclnnis RJ, Southey MC, Nguyen-Dumont T, Cancel-Tassin G, et al: Two-stage Study of Familial Prostate Cancer by Wholeexome Sequencing and Custom Capture Identifies 10 Novel Genes Associated with the Risk of Prostate Cancer. Eur Urol 2020.

23. Wu YQ, Ju CL, Wang BJ, Wang RG: PABPC1L depletion inhibits proliferation and migration via blockage of AKT pathway in human colorectal cancer cells. Oncol Lett 2019, 17:3439-3445.

24. Zhang H, Xu HB, Kurban E, Luo HW: LncRNA SNHG14 promotes hepatocellular carcinoma progression via H3K27 acetylation activated PABPC1 by PTEN signaling. Cell Death Dis 2020, 11:646.

25. Zhang H, Sheng C, Yin Y, Wen S, Yang G, Cheng Z, Zhu Q: PABPC1 interacts with AGO2 and is responsible for the microRNA mediated gene silencing in high grade hepatocellular carcinoma. Cancer Lett 2015, 367:49-57.

26. Zhu J, Ding H, Wang X, Lu Q: 2015 PABPC1 exerts carcinogenesis in gastric carcinoma by targeting miR-34c. Int J Clin Exp Pathol 2015, 8:3794-3802.

\section{Tables}


Table 1

Associations of PABPC1 expression with clinicopathological characteristics in GC

\begin{tabular}{|c|c|c|c|c|c|}
\hline \multirow[t]{2}{*}{ Characteristics } & \multirow{2}{*}{$\begin{array}{l}\text { No. } \\
(N=170)\end{array}$} & \multicolumn{2}{|c|}{ PABPC1 expression } & \multirow{2}{*}{$x^{2}$} & \multirow[t]{2}{*}{$\mathrm{P}$} \\
\hline & & $\operatorname{Low}(\mathrm{N}=79)$ & $\operatorname{High}(\mathrm{N}=91)$ & & \\
\hline Age & & $57.58 \pm 12.22$ & $57.38 \pm 11.88$ & 0.000 & 0.999 \\
\hline$\leq 60 y$ & $99(58.2 \%)$ & $46(58.2 \%)$ & $53(58.2 \%)$ & & \\
\hline$\$ 60 y$ & $71(41.8 \%)$ & $33(41.8 \%)$ & $38(41.8 \%)$ & & \\
\hline Gender & & & & 2.295 & 0.130 \\
\hline Male & $124(72.9 \%)$ & $62(78.5 \%)$ & $62(68.1 \%)$ & & \\
\hline Female & $46(27.1 \%)$ & $17(21.5 \%)$ & $29(31.9 \%)$ & & \\
\hline Tumor size & & & & 6.960 & 0.008 \\
\hline $5 \mathrm{~cm}$ & $121(71.2 \%)$ & $64(81.0 \%)$ & $57(62.6 \%)$ & & \\
\hline$\nabla 5 \mathrm{~cm}$ & $49(28.8 \%)$ & $15(19.0 \%)$ & $34(37.4 \%)$ & & \\
\hline Borrmann classification & & & & 8.666 & 0.003 \\
\hline$\nabla+\square$ & $66(38.8 \%)$ & $40(50.6 \%)$ & $26(28.6 \%)$ & & \\
\hline$\nabla+\square$ & $104(61.2 \%)$ & $39(49.4 \%)$ & $65(71.4 \%)$ & & \\
\hline Differentiation & & & & 0.959 & 0.619 \\
\hline Well & $5(2.9 \%)$ & $3(3.8 \%)$ & $2(2.2 \%)$ & & \\
\hline Moderate & $46(20.1 \%)$ & $19(24.1 \%)$ & $27(29.7 \%)$ & & \\
\hline Poor & $119(70.0 \%)$ & $57(72.1 \%)$ & $62(68.1 \%)$ & & \\
\hline Vessel invasion & & & & 5.743 & 0.017 \\
\hline No & $140(82.4 \%)$ & $71(89.9 \%)$ & $69(75.9 \%)$ & & \\
\hline Yes & $30(17.6 \%)$ & $8(10.1 \%)$ & $22(24.2 \%)$ & & \\
\hline Nerve invasion & & & & 1.141 & 0.285 \\
\hline No & $155(91.2 \%)$ & $74(93.7 \%)$ & $81(89.0 \%)$ & & \\
\hline Yes & $15(8.8 \%)$ & $5(6.3 \%)$ & $10(11.0 \%)$ & & \\
\hline Depth of invasion & & & & 10.588 & 0.032 \\
\hline $\mathrm{T} 1$ & $29(17.1 \%)$ & $19(24.1 \%)$ & $10(11.0 \%)$ & & \\
\hline $\mathrm{T} 2$ & $24(14.1 \%)$ & $10(12.7 \%)$ & $14(15.4 \%)$ & & \\
\hline T3 & $58(34.1 \%)$ & $31(39.2 \%)$ & $27(29.7 \%)$ & & \\
\hline
\end{tabular}




\begin{tabular}{|c|c|c|c|c|c|}
\hline T4a & $38(22.4 \%)$ & $13(16.4 \%)$ & $25(27.4 \%)$ & & \\
\hline $\mathrm{T} 4 \mathrm{~b}$ & $21(12.3 \%)$ & $6(7.6 \%)$ & $15(16.5 \%)$ & & \\
\hline Lymph node metastasis & & & & 19.461 & 0.001 \\
\hline NO & $66(38.8 \%)$ & $38(48.1 \%)$ & $28(30.7 \%)$ & & \\
\hline $\mathrm{N} 1$ & $29(17.1 \%)$ & $15(18.9 \%)$ & $14(15.4 \%)$ & & \\
\hline $\mathrm{N} 2 \mathrm{a}$ & $33(19.4 \%)$ & $18(22.8 \%)$ & $15(16.5 \%)$ & & \\
\hline $\mathrm{N} 2 \mathrm{~b}$ & $24(14.1 \%)$ & $7(8.9 \%)$ & $17(18.7 \%)$ & & \\
\hline N3 & $18(10.6 \%)$ & $1(1.3 \%)$ & $17(18.7 \%)$ & & \\
\hline pTNM & & & & 7.912 & 0.019 \\
\hline प & $43(25.3 \%)$ & $25(31.6 \%)$ & $18(19.8 \%)$ & & \\
\hline प & $45(26.5 \%)$ & $25(31.6 \%)$ & $20(22.0 \%)$ & & \\
\hline$\nabla+\rrbracket$ & $82(48.2 \%)$ & $29(36.8 \%)$ & $53(58.2 \%)$ & & \\
\hline CEA level $(\mu \mathrm{g} / \mathrm{L})$ & & & & 2.181 & 0.105 \\
\hline$\leq 5$ & $148(87.1 \%)$ & 72(91.1\%) & $76(83.5 \%)$ & & \\
\hline$\$ 5$ & $22(12.9 \%)$ & $7(8.9 \%)$ & $15(16.5 \%)$ & & \\
\hline
\end{tabular}


Table 2

Cox proportional-hazard regression analysis for overall survival

\begin{tabular}{|c|c|c|c|c|c|c|c|c|}
\hline \multirow[t]{3}{*}{ Characteristic } & \multicolumn{4}{|c|}{ Univariate analysis } & \multicolumn{4}{|c|}{ Multivariate analysis } \\
\hline & \multirow[t]{2}{*}{$P$} & \multirow[t]{2}{*}{ HR } & \multicolumn{2}{|c|}{$\begin{array}{l}95.0 \% \mathrm{Cl} \text { for } \\
\operatorname{Exp}(\mathrm{B})\end{array}$} & \multirow[t]{2}{*}{$P$} & \multirow[t]{2}{*}{ HR } & \multicolumn{2}{|c|}{$\begin{array}{l}95.0 \% \mathrm{Cl} \text { for } \\
\operatorname{Exp}(\mathrm{B})\end{array}$} \\
\hline & & & Lower & Upper & & & Lower & Upper \\
\hline Gender & 0.058 & 1.623 & 0.983 & 2.680 & & & & \\
\hline Age & 0.603 & 1.131 & 0.710 & 1.802 & & & & \\
\hline Tumor size & 0.021 & 2.464 & 1.543 & 3.935 & & & & \\
\hline $\begin{array}{l}\text { Bornmann } \\
\text { classification }\end{array}$ & $<0.001$ & 4.388 & 2.358 & 8.168 & & & & \\
\hline Differentiation & 0.028 & 1.771 & 1.063 & 2.953 & & & & \\
\hline Depth of invasion & $<0.001$ & 1.874 & 1.519 & 2.313 & 0.048 & 1.400 & 1.001 & 1.958 \\
\hline $\begin{array}{l}\text { Lymph node } \\
\text { metastasis }\end{array}$ & $<0.001$ & 2.290 & 1.887 & 2.779 & 0.012 & 1.796 & 1.342 & 2.405 \\
\hline pTNM & $<0.001$ & 3.620 & 2.369 & 5.531 & 0.027 & 2.875 & 1.969 & 3.319 \\
\hline Vessel invasion & 0.031 & 3.365 & 2.018 & 5.612 & & & & \\
\hline Nerve invasion & 0.018 & 2.522 & 1.278 & 4.977 & & & & \\
\hline CEA & 0.007 & 2.228 & 1.240 & 4.004 & & & & \\
\hline PABPC1 expression & $<0.001$ & 3.643 & 2.149 & 6.176 & 0.013 & 2.058 & 1.162 & 3.644 \\
\hline
\end{tabular}


Table 3

Cox proportional-hazard regression analysis for disease-free survival

\begin{tabular}{|c|c|c|c|c|c|c|c|c|}
\hline \multirow[t]{3}{*}{ Characteristic } & \multicolumn{4}{|c|}{ Univariate analysis } & \multicolumn{4}{|c|}{ Multivariate analysis } \\
\hline & \multirow[t]{2}{*}{$P$} & \multirow[t]{2}{*}{ HR } & \multicolumn{2}{|c|}{$\begin{array}{l}95.0 \% \mathrm{Cl} \text { for } \\
\operatorname{Exp}(\mathrm{B})\end{array}$} & \multirow[t]{2}{*}{$P$} & \multirow[t]{2}{*}{$\mathrm{HR}$} & \multicolumn{2}{|c|}{$\begin{array}{l}95.0 \% \mathrm{Cl} \text { for } \\
\operatorname{Exp}(\mathrm{B})\end{array}$} \\
\hline & & & Lower & Upper & & & Lower & Upper \\
\hline Gender & 0.193 & 1.505 & 0.813 & 2.784 & & & & \\
\hline Age & 0.616 & 0.860 & 0.477 & 1.549 & & & & \\
\hline Tumor size & 0.053 & 2.563 & 1.435 & 4.578 & & & & \\
\hline $\begin{array}{l}\text { Bornmann } \\
\text { classification }\end{array}$ & 0.009 & 3.892 & 1.880 & 8.060 & & & & \\
\hline Differentiation & 0.011 & 2.553 & 1.236 & 5.271 & & & & \\
\hline Depth of invasion & $<0.001$ & 1.858 & 1.442 & 2.395 & & & & \\
\hline $\begin{array}{l}\text { Lymph node } \\
\text { metastasis }\end{array}$ & 0.038 & 2.194 & 1.735 & 2.774 & & & & \\
\hline pTNM & $<0.001$ & 3.761 & 2.230 & 6.342 & 0.021 & 3.874 & 2.649 & 4.434 \\
\hline Vessel invasion & 0.008 & 4.892 & 2.667 & 8.972 & 0.010 & 2.700 & 1.265 & 5.764 \\
\hline Nerve invasion & 0.042 & 2.911 & 1.349 & 6.282 & & & & \\
\hline CEA & 0.044 & 2.115 & 1.021 & 4.380 & & & & \\
\hline PABPC1 expression & 0.008 & 3.450 & 1.813 & 6.564 & 0.018 & 2.284 & 1.153 & 4.524 \\
\hline
\end{tabular}

\section{Figures}




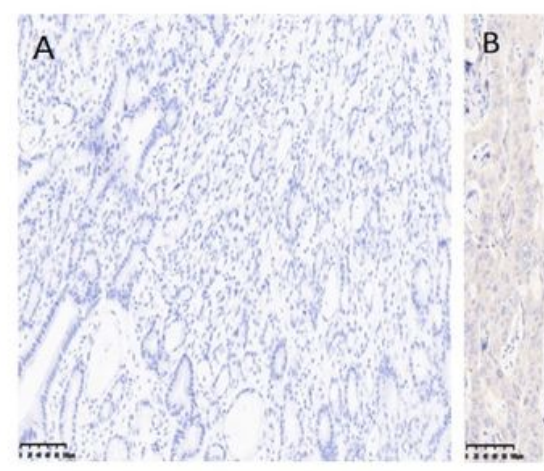

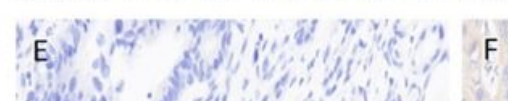

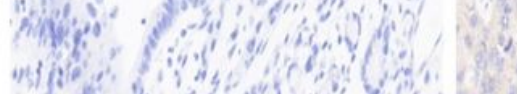

15

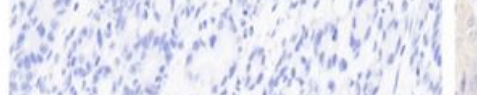

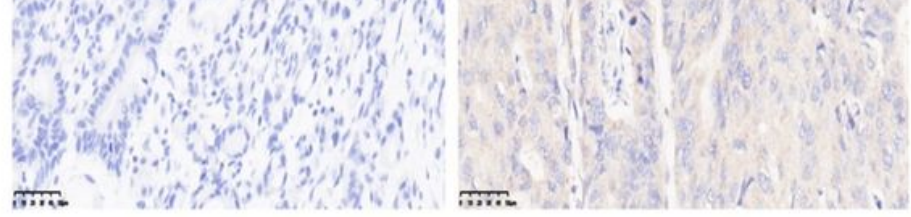

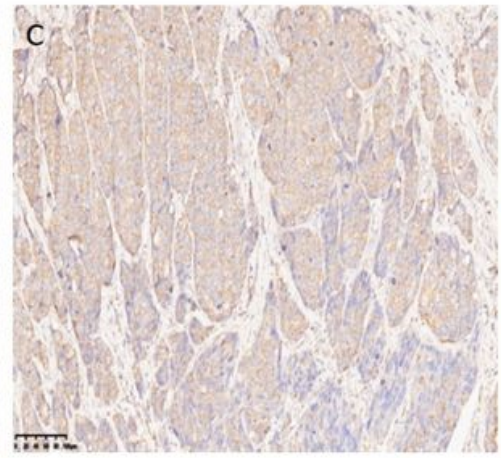
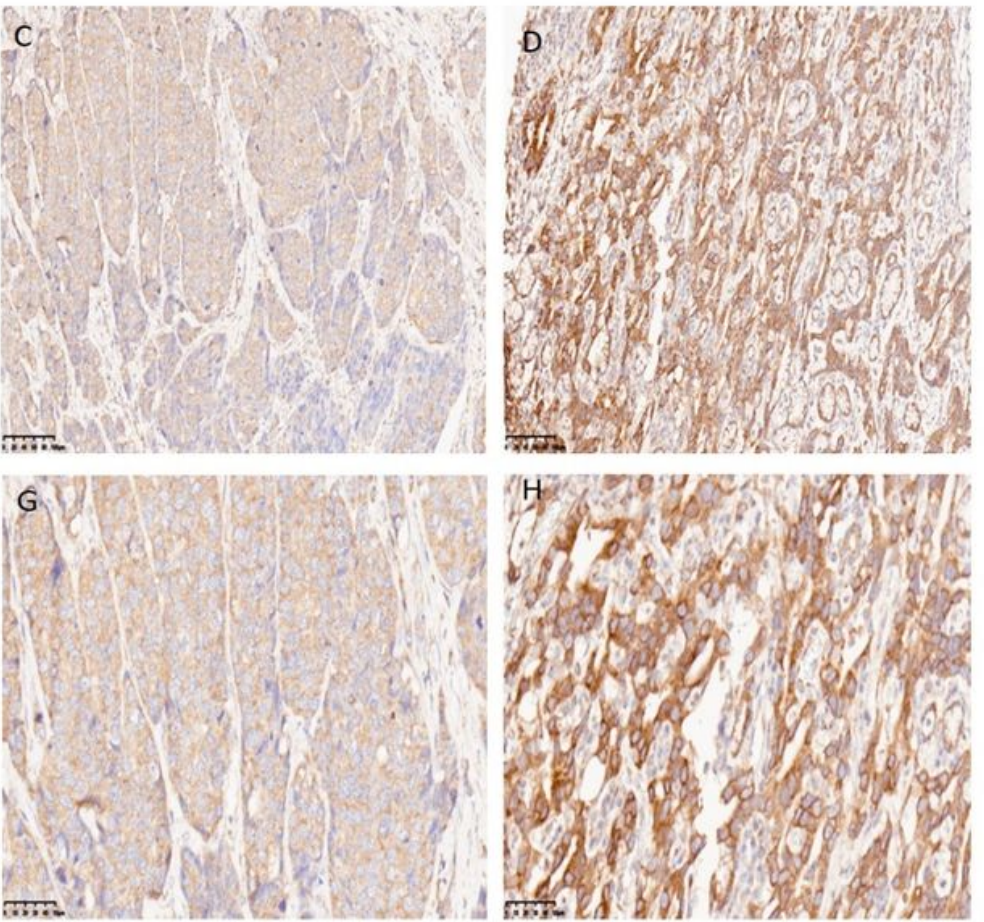

Figure 1

Different expression intensities of PABPC1 in GC tissues assessed by IHC staining. a: No staining at 20x. b: Weak staining at 20x. c: Moderate staining at 20x. d: Strong staining at 20x. e: No staining at 40x. f: Weak staining at 40x. g: Moderate staining at 40x. h: Strong staining at 40x.
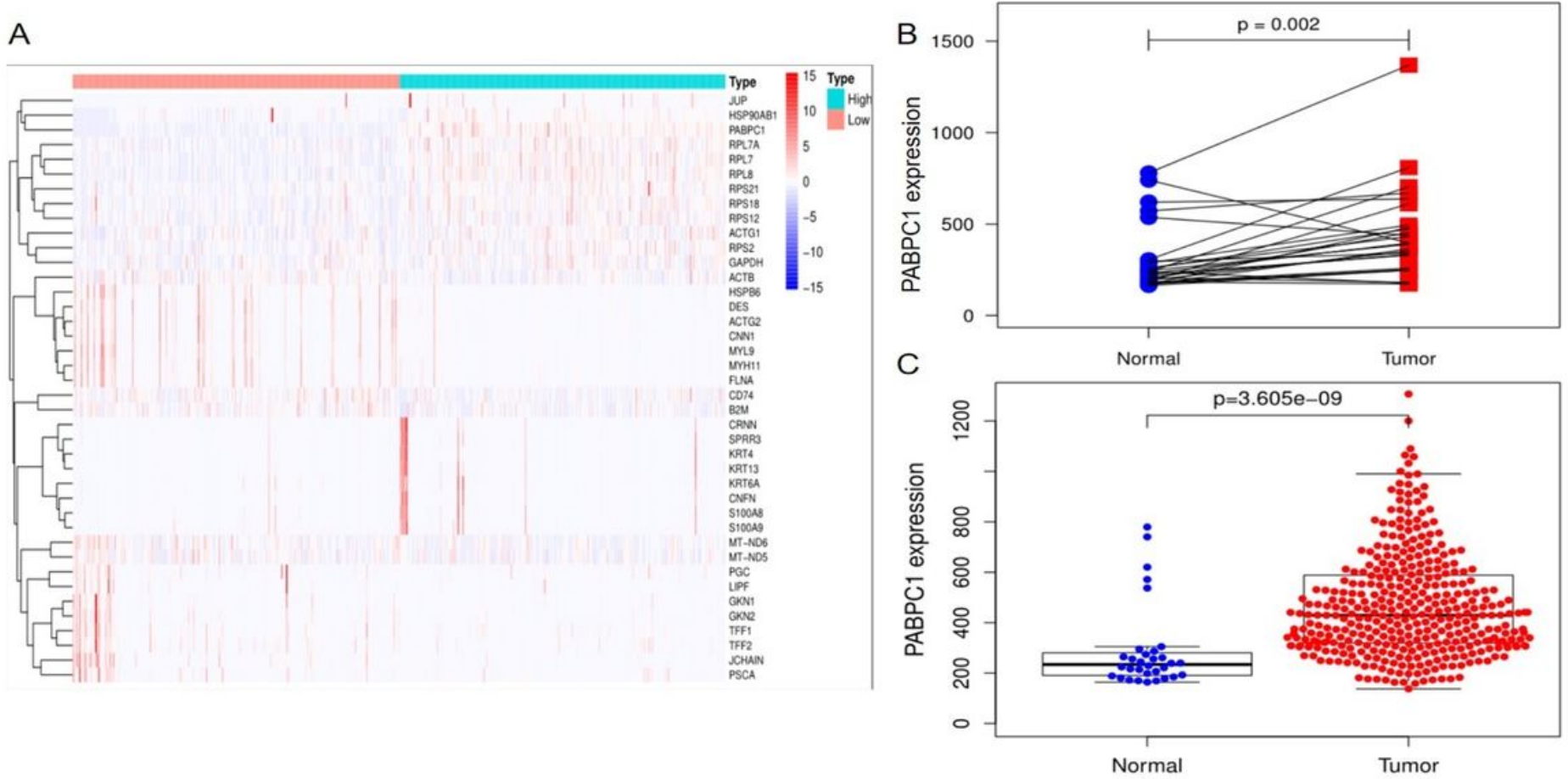

Figure 2 
The expression of PABPC1 in GC according to bioinformatics analysis. a: Analysis of the PABPC1-related data from TCGA database in a heat map. b: The mRNA level of PABPC1 in paired GC and normal gastric tissues from TCGA database. c: The mRNA level of PABPC1 in unpaired GC and normal gastric tissues from TCGA database.

A

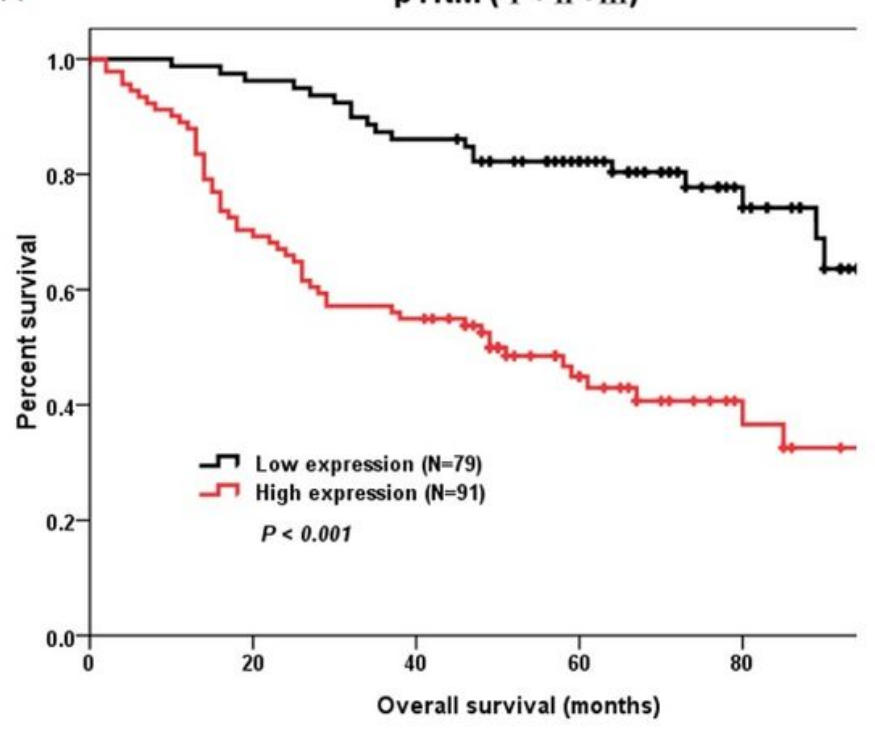

B

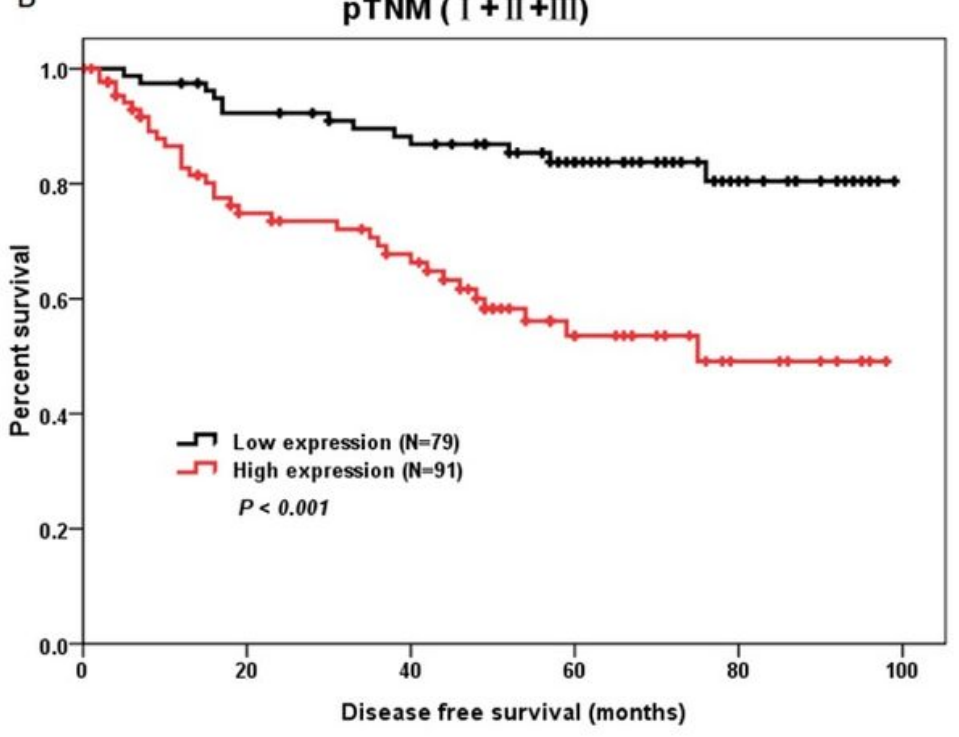

\section{Figure 3}

Impact of PABPC1 on overall survival and disease-free survival a: Patients with high PABPC1 expression had worse overall survival. b: Patients with high PABPC1 expression had worse disease-free survival. 